\title{
Towards a Typed Geometry of Interaction
}

\author{
Esfandiar Haghverdi ${ }^{1}$ and Philip J. Scott ${ }^{2}$ \\ 1 School of Informatics, Indiana University \\ Bloomington, IN 47406, USA, \\ ehaghver@indiana.edu, \\ WWW homepage: http://xavier.informatics.indiana.edu/ ehaghver \\ ${ }^{2}$ Dept. of Mathematics \& Statistics \\ University of Ottawa, Ottawa, Ontario \\ Canada K1N 6N5, \\ phil@mathstat.uottawa.ca, \\ WWW homepage: http://www.uottawa.ca/site/ phil
}

\begin{abstract}
We introduce a typed version of Girard's Geometry of Interaction, called Multiobject GoI (MGoI) semantics. We give an MGoI interpretation for multiplicative linear logic (MLL) without units which applies to new kinds of models, including finite dimensional vector spaces. For MGoI (i) we develop a version of partial traces and trace ideals (related to previous work of Abramsky, Blute, and Panangaden); (ii) we do not require the existence of a reflexive object for our interpretation (the original GoI 1 and 2 were untyped and hence involved a bureaucracy of domain equation isomorphisms); (iii) we introduce an abstract notion of orthogonality (related to work of Hyland and Schalk) and use this to develop a version of Girard's theory of types, datum and algorithms in our setting, (iv) we prove appropriate Soundness and Completeness Theorems for our interpretations in partially traced categories with orthogonality; (v) we end with an application to completeness of (the original) untyped GoI in a unique decomposition category.
\end{abstract}

\section{Introduction}

Geometry of Interaction (GoI) is a novel kind of semantics, introduced by Girard in a fundamental series of papers beginning in the late 80 's $[13,12,14]$. One of the goals was to provide a mathematical analysis of the dynamics of proof normalization (cut-elimination) independently of logical syntax. To this end, Girard introduced his Execution Formula, a power series representing a dynamical invariant for cut-elimination. Girard's framework, based on $\mathrm{C}^{*}$-algebras, was studied in detail in several works of Danos and Regnier (for example in [9]) and by Malacaria and Regnier [27]. The GoI program has been applied to the analysis of optimal reduction by Gonthier, Abadi, and Lévy [10], to complexity theory [7], to game semantics and token machines [6,25], etc.

Categorical foundations of GoI were initiated in the 90's in lectures by M. Hyland and by S. Abramsky. An early categorical framework was given in [5]. 
Recent work has stressed the role of Joyal-Street-Verity's traced monoidal categories [24] (with additional structure). For example, Abramsky's GoI situations $[1,15,3]$ provide a basic algebraic foundation for GoI for multiplicative, exponential linear logic (MELL). Recently, we used a special kind of GoI situation (with traced unique decomposition categories) to axiomatize the details of Girard's original GoI 1 paper [18].

In our previous papers, we emphasized several important aspects of Girard's seminal work (at least in GoI 1 and 2).

1. The original Girard framework is essentially untyped: there is a reflexive object $U$ in the underlying model (with various retractions and/or domain isomorphisms, e.g. $U \otimes U \triangleleft U)$. Proofs are interpreted in the monoid $H o m(U, U)$.

2. Cut-elimination is interpreted by feedback, hence the use of traced monoidal categories. The Execution Formula, defined via trace, provides an invariant for cut-elimination.

3. Girard introduced an orthogonality operation $\perp$ on endomaps of $U$ together with the notion of types (as sets of endomaps equal to their biorthogonal).

4. There are notions of data and algorithm encoded into this dynamical setting, with fundamental connections between types, algorithms, and the Execution Formula.

Points (1) and (2) above were already emphasized in the Abramsky program, as well as in the work of Danos and Regnier [1,3,18,9]. Orthogonalities have been studied abstractly by Hyland and Schalk [22]. The points (1)-(4) are critical to our view of GoI in $[18,19]$.

Unfortunately, Girard's original GoI is not without its own share of bureaucracy. There are domain isomorphisms (of the reflexive object $U$ ) and an associated $*$-algebra of codings and uncodings. On the one hand, this means the original GoI interpretation of proofs is essentially untyped (i.e. categorically, proofs are interpreted in $\operatorname{Hom}(U, U)$, using the above-mentioned algebra) (see $[3,18, ?])$. On the other hand, this led Danos and Regnier ([9]) to study this algebra in detail in certain concrete models, leading to their extensive analysis of reduction paths in untyped lambda calculus.

Our aim in this paper is to move away from "uni-object GoI" to a typed version. This permits us to both generalize GoI and axiomatize its essential features. For example, by removing reflexive objects $U$, we also unlock the possibilities of generalizing Girard-style GoI to more general tensor categories including cases where the tensor is "product-like" in addition to "sum-like".

The notion of categorical (parametrized) trace was introduced by Joyal, Street and Verity in an influential paper [24]. The motivation for their work arose in algebraic topology and knot theory, although after its publication, it became clear that categorical trace also has many applications in Computer Science, where it includes such notions as feedback, fixedpoints, iteration theories, and so forth. For some history, see $[3,18]$.

In this paper we go one step further and look at partial traces. The idea of generalizing the abstract trace of [24] to the partial setting is not new. For example, partial traces are studied in work of Abramsky, Blute, and Panangaden 
[2] and in unpublished lecture notes of Gordon Plotkin [28]. The guiding example in [2] is the relationship between trace class operators on a Hilbert space and Hilbert-Schmidt operators. This allows the authors to establish a close correspondence between trace and nuclear ideals in a tensor $*$-category. Plotkin's work develops a theory of Conway ideals on biproduct categories, and an associated categorical trace theory. In this paper, we develop a theory of trace ideals sufficient for GoI.

The contributions of this paper can be summarized as follows:

- We introduce an axiomatization for partially traced symmetric monoidal categories and provide examples based on $\mathbf{V e c}_{\mathbf{f d}}$, finite dimensional vector spaces, and CMet, complete metric spaces. We also provide illuminating non-examples. Our axiomatization is different from that in [2], although related in spirit.

- We introduce an abstract orthogonality [22], appropriate for GoI, on our models.

- We introduce a multiobject version of Girard's GoI semantics (MGoI) in partially traced models with orthogonality, including Girard's notions of types, datum, algorithm and the execution formula. We give an MGoI interpretation for the multiplicative fragment of linear logic without units (MLL) and show that the execution formula is an invariant of cut-elimination (see Section 5 below). Note that Girard's original GoI (as presented in [3] ) requires a reflexive object $U \neq\{\mathbf{0}\}$, i.e. a retraction $U \oplus U \triangleleft U$, which is impossible in $\mathbf{V e c}_{\mathrm{fd}}$.

- We prove a soundness and completeness theorem for our MGoI interpretation of MLL in arbitrary partially traced categories with orthogonality relation. As an application, we also prove a completeness result for untyped GoI semantics of MLL in a traced UDC based GoI Situation.

The rest of the paper is organized as follows. In Section 2 we introduce partially traced symmetric monoidal categories and discuss some examples. In Section 3 we introduce the abstract orthogonality relation in a partially traced symmetric monoidal category and discuss how it relates to the work in [22]. In Section 4 we introduce our new semantics, MGoI, and give an interpretation for MLL. Section 5 discusses the execution formula and the soundness theorem, while in Section 6 we prove a completeness theorem for the MGoI interpretation of MLL in a partially traced category with an orthogonality relation. As an application, in Section 7 we prove a completeness result for untyped GoI semantics of MLL in a traced UDC based GoI Situation. Finally, Section 8 contains some thoughts about possible future directions, projects and links to related work in the literature.

Note: The full proofs of the results will appear in the journal version.

\section{Trace Ideals}

We now give an axiomatization for partial parametric trace, or partial trace for short, suitable for our purposes. Recall, following Joyal, Street, and Verity [24], 
a parametric trace in a symmetric monoidal category $(\mathbb{C}, \otimes, I, s)$ is an operator $\operatorname{Tr}_{X, Y}^{U}: \mathbb{C}(X \otimes U, Y \otimes U) \rightarrow \mathbb{C}(X, Y)$, satisfying various well-known naturality equations. A partial parametric trace requires instead that $\operatorname{Tr}_{X, Y}^{U}$ be a partial map. The domain of this map, denoted $\mathbb{T}_{X, Y}^{U}$, is called a trace ideal and must satisfy various closure conditions.

Definition 1 (Trace Ideal). Let $(\mathbb{C}, \otimes, I, s)$ be a symmetric monoidal category. $A$ parametric trace ideal in $\mathbb{C}$ is a family of subsets, for each object $U$ of $\mathbb{C}$, of the form

$$
\mathbb{T}_{X, Y}^{U} \subseteq \mathbb{C}(X \otimes U, Y \otimes U) \text { for all objects } X, Y \text { of } \mathbb{C}
$$

together with a family of operations, called a parametric partial trace, of the form

$$
\operatorname{Tr}_{X, Y}^{U}: \mathbb{T}_{X, Y}^{U} \rightarrow \mathbb{C}(X, Y)
$$

subject to the following axioms. Here the parameters are $X$ and $Y$ and a morphism $f \in \mathbb{T}_{X, Y}^{U}$ is said to be of trace class.

- Naturality in $X$ and $Y:$ For any $f \in \mathbb{T}_{X, Y}^{U}$ and $g: X^{\prime} \rightarrow X$ and $h: Y \rightarrow Y^{\prime}$,

$$
\left(h \otimes 1_{U}\right) f\left(g \otimes 1_{U}\right) \in \mathbb{T}_{X^{\prime}, Y^{\prime}}^{U}
$$

and

$$
\operatorname{Tr}_{X^{\prime}, Y^{\prime}}^{U}\left(\left(h \otimes 1_{U}\right) f\left(g \otimes 1_{U}\right)\right)=h \operatorname{Tr}_{X, Y}^{U}(f) g
$$

- Dinaturality in $U:$ For any $f: X \otimes U \rightarrow Y \otimes U^{\prime}, g: U^{\prime} \rightarrow U$,

$$
\left(1_{Y} \otimes g\right) f \in \mathbb{T}_{X, Y}^{U} \quad \text { iff } f\left(1_{X} \otimes g\right) \in \mathbb{T}_{X, Y}^{U^{\prime}},
$$

and

$$
\operatorname{Tr}_{X, Y}^{U}\left(\left(1_{Y} \otimes g\right) f\right)=\operatorname{Tr}_{X, Y}^{U^{\prime}}\left(f\left(1_{X} \otimes g\right)\right) .
$$

- Vanishing $\mathbf{I}: \mathbb{T}_{X, Y}^{I}=\mathbb{C}(X, Y)$ and for $f \in \mathbb{T}_{X, Y}^{I}$

$$
\operatorname{Tr}_{X, Y}^{I}(f)=f
$$

- Vanishing II: For any $g: X \otimes U \otimes V \rightarrow Y \otimes U \otimes V$, if $g \in \mathbb{T}_{X \otimes U, Y \otimes U}^{V}$, then

$$
g \in \mathbb{T}_{X, Y}^{U \otimes V} \text { iff } \operatorname{Tr}_{X \otimes U, Y \otimes U}^{V}(g) \in \mathbb{T}_{X, Y}^{U}
$$

and

$$
\operatorname{Tr}_{X, Y}^{U \otimes V}(g)=\operatorname{Tr}_{X, Y}^{U}\left(\operatorname{Tr}_{X \otimes U, Y \otimes U}^{V}(g)\right) .
$$

- Superposing: For any $f \in \mathbb{T}_{X, Y}^{U}$ and $g: W \rightarrow Z$,

$$
g \otimes f \in \mathbb{T}_{W \otimes X, Z \otimes Y}^{U}
$$

and

$$
\operatorname{Tr}_{W \otimes X, Z \otimes Y}^{U}(g \otimes f)=g \otimes \operatorname{Tr}_{X, Y}^{U}(f)
$$


- Yanking: $\quad s_{U U} \in \mathbb{T}_{U, U}^{U}, \quad$ and $\operatorname{Tr}_{U, U}^{U}\left(s_{U, U}\right)=1_{U}$.

A monoidal category $(\mathbb{C}, \otimes, I, s)$ with such a trace ideal is called a partially traced category. In the case $X$ and $Y$ are chosen to be $I$ (the unit of the tensor), we say this defines a nonparametric trace.

Remark 1. As we mentioned earlier, the first definition of a partial parametric trace is due to Abramsky, Blute and Panangaden in [2]. Our definition is different but related to theirs. First, we have used the Yanking axiom in Joyal, Street and Verity [24], whereas in [2] they use so-called "generalized yanking"; that is, for $f: X \rightarrow U$ and $g: U \rightarrow Y, \operatorname{Tr}_{X, Y}^{U}\left(s_{U, Y}(f \otimes g)\right)=g f$ whenever $s_{U, Y}(f \otimes g)$ is of trace class. It was shown in [15] that for traced monoidal categories the two axioms of yanking and generalized yanking are equivalent in the presence of all the other axioms. This equivalence remains valid for the partially traced categories introduced here. In our examples $s_{U U}$ is traceable for all $U$; on the other hand, many examples in [2] do not have this property. Our Vanishing II axiom differs from and is weaker than the one proposed in [2]. More importantly, we do not require one of the ideal axioms in [2]. Namely, we do not ask that for $f \in \mathbb{T}_{X, Y}^{U}$ and any $h: U \rightarrow U,\left(1_{Y} \otimes h\right) f$ and $f\left(1_{X} \otimes h\right)$ be in $\mathbb{T}_{X, Y}^{U}$. Indeed in the next section we prove that the categories $\left(\mathbf{V e c}_{\mathbf{f d}}, \oplus\right)$ of finite dimensional vector spaces, and $(\mathbf{C M e t}, \times)$ of complete metric spaces are partially traced. It can be shown that in both categories the above ideal axiom and vanishing II of [2] fail and hence they are not traced in the sense of ABP. In defense of not enforcing this ideal axiom, we observe that it is not required for any of the trace axioms. Any partially traced category in the sense of ABP for which the yanking axiom holds will be partially traced according to our definition.

By letting the parameters $X$ and $Y$ be the unit I of the tensor, we get the nonparametric version of our partial trace, which is again different from the one in [2].

\section{$2.1 \quad$ Examples}

\section{(a) Finite Dimensional Vector Spaces}

The category $\mathbf{V e c}_{\mathbf{f d}}$ of finite dimensional vector spaces and linear transformations is a symmetric monoidal, indeed an additive, category (see [26]), with monoidal product taken to be $\oplus$, the direct sum (biproduct). Hence, given $f: \oplus_{I} X_{i} \rightarrow \oplus_{J} Y_{j}$ with $|I|=n$ and $|J|=m$, we can write $f$ as an $m \times n$ matrix $f=\left[f_{i j}\right]$ of its components, where $f_{i j}: X_{j} \rightarrow Y_{i}$ (notice the switch in the indices $i$ and $j$ ).

We give a trace ideal structure on the category $\left(\mathbf{V e c}_{\mathbf{f d}}, \oplus, \mathbf{0}\right)$ as follows. We shall say an $f: X \oplus U \rightarrow Y \oplus U$ is of trace class iff $\left(I-f_{22}\right)$ is invertible, where $I$ and $f_{22}$ have size $\operatorname{dim}(U)$. In that case, we write

$$
\operatorname{Tr}_{X, Y}^{U}(f)=f_{11}+f_{12}\left(I-f_{22}\right)^{-1} f_{21} .
$$


Proposition 1. $\left(\mathbf{V e c}_{\mathbf{f d}}, \oplus, \mathbf{0}\right)$ is a partially traced category with trace ideal as above.

We shall need the following standard results for the proof of this proposition.

Lemma 1. Let $M=\left[\begin{array}{ll}A & B \\ C & D\end{array}\right]$ be a partitioned matrix with blocks $A(m \times m)$, $B(m \times n), C(n \times m)$ and $D(n \times n)$. If $D$ is invertible, then $M$ is invertible iff $A-B D^{-1} C$ is invertible.

Lemma 2. Given $A(m \times n)$ and $B(n \times m),\left(I_{m}-A B\right)$ is invertible iff $\left(I_{n}-B A\right)$ is invertible. Moreover $\left(I_{m}-A B\right)^{-1} A=A\left(I_{n}-B A\right)^{-1}$.

\section{(b) Other Finite Dimensional Examples}

The proof of Proposition 1 remains valid for the category $\left(\mathbf{H i l b}_{\mathbf{f d}}, \oplus\right)$ of finite dimensional Hilbert spaces (under $\oplus$ ) and bounded linear maps. As discussed in Remark 1, the category $\left(\mathbf{V e c}_{\mathbf{f d}}, \oplus\right.$ ) is not partially traced in the sense of ABP.

\section{(c) Metric Spaces}

Consider the category CMet of complete metric spaces with non-expansive maps. Define $f:\left(M, d_{M}\right) \rightarrow\left(N, d_{N}\right)$ to be non-expansive iff there is a fixed $0 \leq \alpha \leq 1$ such that $d_{N}(f(x), f(y)) \leq \alpha d_{M}(x, y)$ for all $x, y \in M$. The greatest lower bound of such $\alpha$ is called the Lipschitz constant of $f$. Note that the tempting collection of complete metric spaces and contractions $(\alpha<1)$ is not a category: there are no identity morphisms! CMet has products, namely given $\left(M, d_{M}\right)$ and $\left(N, d_{N}\right)$ we define $\left(M \times N, d_{M \times N}\right)$ with $d_{M \times N}\left((m, n),\left(m^{\prime}, n^{\prime}\right)\right)=$ $\max \left\{d_{M}\left(m, m^{\prime}\right), d_{N}\left(n, n^{\prime}\right)\right\}$.

We define the trace structure on CMet (where $\otimes=\times$ ) as follows. We say that a morphism $f: X \times U \rightarrow Y \times U$ is in $\mathbb{T}_{X, Y}^{U}$ iff $\pi_{2} \lambda u . f(x, u): U \rightarrow U$ has a unique fixed point for every $x \in X$, in other words iff for every $x \in X$, there is a unique $u$ such that $f(x, u)=(y, u)$, for some $y$. Note that contractions have unique fixed points, by the Banach fixed point theorem.

Suppose $f \in \mathbb{T}_{X, Y}^{U}$. We define $\operatorname{Tr}_{X, Y}^{U}(f): X \rightarrow Y$ by $\operatorname{Tr}_{X, Y}^{U}(f)(x)=y$, where $f(x, u)=(y, u)$ for the unique $u$. Equivalently, $\operatorname{Tr}_{X, Y}^{U}(f)(x)=\pi_{1} f(x, u)$ where $u$ is the unique fixed point of $\pi_{2} \lambda t . f(x, t)$.

Proposition 2. (CMet,$\times,\{*\})$ is a partially traced category with trace ideal as above.

Lemma 3. Let $A$ and $B$ be two complete metric spaces and $f: A \rightarrow B$ and $g: B \rightarrow A$. Then, $g f$ has a unique fixed point if and only if $f g$ does. Moreover, let $a \in A$ be the unique fixed point of $g f: A \rightarrow A$ and $b \in B$ be the unique fixed point of $f g: B \rightarrow B$. Then $f(a)=b$ and $g(b)=a$. 
Note that the proof above does not use the properties of a metric space; hence, the proof remains valid for the category (Sets, $\times$ ) of sets and mappings. The latter then becomes a partially traced category with the same definition for trace class morphisms as in CMet. However, this fails for the category $(\mathbf{R e l}, \times)$, of sets and relations: consider the sets $A=\{a\}, B=\left\{b, b^{\prime}\right\}$, and let $f=\left\{(a, b),\left(a, b^{\prime}\right)\right\}$ and $g=\left\{(b, a),\left(b^{\prime}, a\right)\right\}$.

\section{(d) Total Traces}

Of course, all (totally-defined) traces in the usual definition of a traced monoidal category yield a trace ideal, namely the entire homset is the domain of $\mathrm{Tr}$. In particular, all the examples in our previous work on uni-object GoI $[18,19]$, for example based on unique decomposition categories, still apply here.

\section{(e) Some Non-Examples}

Let $\mathcal{M}$ be some class of morphisms of appropriate arity in CMet. We wish to form trace ideals on the structure $(\mathbf{C M e t}, \times, \mathcal{M})$. Obviously, this depends on a good theory of fixed points as we saw in the preceding example. However, defining the trace class morphisms as $f$ such that $\pi_{2} \lambda u . f(x, u): U \rightarrow U$ is a contraction for every $x \in X$, does not yield a partially traced category: all axioms are true except for dinaturality and vanishing II. Thus, the collection $\mathcal{M}$ $=$ all contractions fails to be a trace ideal in our sense.

This suggests an oriented equality on some of the equations of a trace (e.g. Vanishing II) and an alteration of the dinaturality axiom. This direction has been pursued by Plotkin [28]. This approach fails for us, since one-sided (directed) equations cause problems in applications to GoI. Given the well-developed theory of fixed points in Banach Spaces, we hope to still find more examples of trace classes in various categories of such spaces.

\section{Orthogonality Relations}

Girard originally introduced orthogonality relations into linear logic to model formulas (or types) (e.g. in the phase semantics of the original paper [11] and in GoI 1 [12]). Recently Hyland and Schalk gave an abstract approach to orthogonality relations in symmetric monoidal closed categories [22]. They also point out that an orthogonality on a traced symmetric monoidal category can be obtained by considering their axioms applied to $\operatorname{Int}(\mathbb{C})$ and then translating them down to $\mathbb{C}$. Below we give this translation (not explicitly calculated in $[22]$ ), using the category $\mathcal{G}(\mathbb{C})[1,15]$ instead of $\operatorname{Int}(\mathbb{C})$. The categories $\mathcal{G}(\mathbb{C})$ and $\operatorname{Int}(\mathbb{C})$ are shown to be isomorphic in $[15]$. As we are dealing with partial trace we need to take extra care in stating the axioms below, namely that an axiom involving a trace should be read with the proviso: "whenever all traces exist".

Definition 2. Let $\mathbb{C}$ be a traced symmetric monoidal category. An orthogonality relation on $\mathbb{C}$ is a family of relations $\perp_{U V}$ between maps $u: V \rightarrow U$ and $x$ : $U \rightarrow V$

$$
V \stackrel{u}{\longrightarrow} U \perp_{U V} U \stackrel{x}{\longrightarrow} V
$$


subject to the following axioms:

(i) Isomorphism: Let $f: U \otimes V^{\prime} \rightarrow V \otimes U^{\prime}$ and $f^{-1}: U^{\prime} \otimes V \rightarrow V^{\prime} \otimes U$ be such that $\left.\operatorname{Tr}^{V^{\prime}}\left(\operatorname{Tr}^{U^{\prime}}\left(\left(1 \otimes 1 \otimes s_{U^{\prime}, V^{\prime}}\right) \alpha^{-1}\left(f \otimes f^{-1}\right) \alpha\right)\right)\right)=s_{U, V}$ and $\operatorname{Tr}^{V}\left(\operatorname{Tr}^{U}((1 \otimes\right.$ $\left.\left.\left.\left.1 \otimes s_{U, V}\right) \alpha^{-1}\left(f^{-1} \otimes f\right) \alpha\right)\right)\right)=s_{U^{\prime}, V^{\prime}}$. Here $\alpha=(1 \otimes 1 \otimes s)(1 \otimes s \otimes 1)$ with $s$ at appropriate types. Note that this simply means that $f:(U, V) \rightarrow\left(U^{\prime}, V^{\prime}\right)$ and $f^{-1}:\left(U^{\prime}, V^{\prime}\right) \rightarrow(U, V)$ are inverses of each other in $\mathcal{G}(\mathbb{C})$.

Then for all $u: V \rightarrow U$ and $x: U \rightarrow V$,

$$
u \perp_{U V} x \text { iff } \operatorname{Tr}_{V^{\prime}, U^{\prime}}^{U}\left(s_{U, U^{\prime}}\left(u \otimes 1_{U^{\prime}}\right) f s_{V^{\prime}, U}\right) \perp_{U^{\prime} V^{\prime}} \operatorname{Tr}_{U^{\prime}, V^{\prime}}^{V}\left(\left(1_{V^{\prime}} \otimes x\right) f^{-1}\right)
$$

(ii) Tensor: For all $u: V \rightarrow U, v: V^{\prime} \rightarrow U^{\prime}$ and $h: U \otimes U^{\prime} \rightarrow V \otimes V^{\prime}$,

$$
\begin{gathered}
u \perp_{U V} \operatorname{Tr}_{U, V}^{U^{\prime}}\left(\left(1_{V} \otimes v\right) h\right) \text { and } v \perp_{U^{\prime} V^{\prime}} \operatorname{Tr}_{U^{\prime}, V^{\prime}}^{U}\left(s_{U, V^{\prime}}\left(u \otimes 1_{V^{\prime}}\right) h s_{U^{\prime}, U}\right) \\
\text { implies } \\
(u \otimes v) \perp_{U \otimes U^{\prime}, V \otimes V^{\prime}} h
\end{gathered}
$$

(iii) Implication: For all $u: V \rightarrow U, y: U^{\prime} \rightarrow V^{\prime}$ and $f: U \otimes V^{\prime} \rightarrow V \otimes U^{\prime}$

$$
\begin{gathered}
u \perp_{U V} \operatorname{Tr}_{U, V}^{V^{\prime}}\left(\left(1_{V} \otimes y\right) f\right) \text { and } \operatorname{Tr}_{V^{\prime}, U^{\prime}}^{V}\left(s_{V, U^{\prime}} f\left(u \otimes 1_{V^{\prime}}\right) s_{V^{\prime}, V}\right) \perp_{U^{\prime} V^{\prime}} y \\
\\
\quad \text { implies } \\
f \perp_{V \otimes U^{\prime}, U \otimes V^{\prime}}(u \otimes y)
\end{gathered}
$$

(iv) Identity: For all $u: V \rightarrow U$ and $x: U \rightarrow V$

$$
u \perp_{U V} x \text { implies } 1_{I} \perp_{I I} \operatorname{Tr}_{I, I}^{V}(x u)
$$

(v) Symmetry: For all $u: V \rightarrow U$ and $x: U \rightarrow V$

$$
u \perp_{U V} x \text { iff } x \perp_{V U} u
$$

Remark 2.

1. It should be noted that for a (partially) traced symmetric monoidal category, the axioms for Tensor and Implication are equivalent in the presence of the other axioms: by dinaturality of trace we have $\operatorname{Tr}_{V^{\prime}, U^{\prime}}^{V}\left(s_{V, U^{\prime}} f(u \otimes\right.$ $\left.\left.\left.1_{V^{\prime}}\right) s_{V^{\prime}, V}\right)=\operatorname{Tr}_{V^{\prime}, U^{\prime}}^{U}\left(s_{U, U^{\prime}}\left(u \otimes 1_{U^{\prime}}\right) f s_{V^{\prime}, U}\right)\right)$, then use the Symmetry axiom. Thus we shall drop the Implication axiom.

2. Our work on GoI reveals that one needs another axiom which we observe as the converse of the Tensor axiom and relaxation of one of the premises, this is related to abstract computation and the notion of datum in GoI. Hence, we shall replace the Tensor axiom by the following the Strong Tensor axiom:

Strong Tensor: For all $u: V \rightarrow U, v: V^{\prime} \rightarrow U^{\prime}$ and $h: U \otimes U^{\prime} \rightarrow V \otimes V^{\prime}$,

$$
v \perp_{U^{\prime} V^{\prime}} \operatorname{Tr}_{U^{\prime}, V^{\prime}}^{U}\left(s_{U, V^{\prime}}\left(u \otimes 1_{V^{\prime}}\right) h s_{U^{\prime}, U}\right) \quad \text { iff } \quad(u \otimes v) \perp_{U \otimes U^{\prime}, V \otimes V^{\prime}} h,
$$

whenever the trace exists. It can be shown that in the presence of the Strong Tensor, Isomorphism, and Symmetry axioms, $v \perp_{U^{\prime} V^{\prime}} \operatorname{Tr}_{U^{\prime}, V^{\prime}}^{U}\left(s_{U, V^{\prime}}(u \otimes\right.$ $\left.\left.1_{V^{\prime}}\right) h s_{U^{\prime}, U}\right)$ implies $u \perp_{U V} \operatorname{Tr}_{U, V}^{U^{\prime}}\left(\left(1_{V} \otimes v\right) h\right)$, whenever all traces exist. 
Definition 3. Let $\mathbb{C}$ be a traced symmetric monoidal category. A strong orthogonality relation is defined as in Definition 2 but with the Tensor Axiom replaced by the Strong Tensor Axiom above, and the Implication axiom dropped.

In the context of GoI, we will be working with strong orthogonality relations on endomorphism sets of objects in the underlying categories. Biorthogonally closed (i.e. $X=X^{\perp \perp}$ ) subsets of certain endomorphism sets are important as they define types (GoI interpretation of formulae.) We have observed that all the orthogonality relations that we work with in this paper can be characterized using trace classes. This suggests the following, which seems to cover many known examples.

Example 1 (Orthogonality as trace class) Let $(\mathbb{C}, \otimes, I, T r)$ be a partially traced category where $\otimes$ is the monoidal product with unit $I$, and $T r$ is the partial trace operator as in Section 2. Let $A$ and $B$ be objects of $\mathbb{C}$. For $f: A \rightarrow B$ and $g: B \rightarrow A$, we can define an orthogonality relation by declaring $f \perp_{B A} g$ iff $g f \in \mathbb{T}_{I, I}^{A}$.

Proof (of Example 1): See the Appendix.

Hence, from our previous discussion on traces, we obtain the following examples:

- $\mathbf{V e c}_{\mathbf{f d}}$. Let $A \in \mathbf{V e c}_{\mathbf{f d}}$. For $f, g \in \operatorname{End}(A)$, define $f \perp g$ iff $I-g f$ is invertible.

- CMet. Let $M \in$ CMet. For $f, g \in \operatorname{End}(M)$, define $f \perp g$ iff $g f$ has a unique fixed point.

\section{Multi-object GoI Interpretation}

In this section we introduce the multiobject geometry of interaction (MGoI) semantics for MLL in a partially traced symmetric monoidal category $(\mathbb{C}, \otimes, I, T r, \perp$ ) equipped with an orthogonality relation $\perp$ as in the previous section. Here $\otimes$ is the monoidal product with unit $I$ and $T r$ is the partial trace operator as in Section 2. We shall point out once again that we do not require that the category $\mathbb{C}$ have a reflexive object, so uni-object GoI semantics may not be possible to carry out in $\mathbb{C}$.

\section{Interpreting formulae:}

Let $A$ be an object of $\mathbb{C}$ and let $f, g \in \operatorname{End}(A)$. We say that $f$ is orthogonal to $g$, denoted $f \perp g$, if $(f, g) \in \perp$. Also given $X \subseteq \operatorname{End}(A)$ we define $X^{\perp}=$ $\{f \in \operatorname{End}(A) \mid f \perp g \forall g \in X\}$. We now define an operator on the objects of $\mathbb{C}$ as follows: Given an object $A, \mathcal{T}(A)=\left\{X \subseteq \operatorname{End}(A) \mid X^{\perp \perp}=X\right\}$. We shall also need the notion of usual denotational interpretation of the formulas. We define an interpretation map \-】 on the formulas of MLL as follows. Given the value of $\llbracket-\rrbracket$ on the atomic propositions as objects of $\mathbb{C}$, we extend it to all formulas by: 


$$
\begin{aligned}
& -\llbracket A^{\perp} \rrbracket=\llbracket A \rrbracket \\
& -\llbracket A \otimes 8 \quad B \rrbracket=\llbracket A \otimes B \rrbracket=\llbracket A \rrbracket \otimes \llbracket B \rrbracket .
\end{aligned}
$$

We then define the MGoI-interpretation for formulas as follows. We use the notation $\theta(A)$ for this interpretation.

- $\theta(\alpha) \in \mathcal{T}(\llbracket \alpha \rrbracket)$, where $\alpha$ is an atomic formula.

$-\theta\left(\alpha^{\perp}\right)=\theta(\alpha)^{\perp}$

- $\theta(A \otimes B)=\{a \otimes b \mid a \in \theta(A), b \in \theta(B)\}^{\perp \perp}$

- $\theta(A>8)=\left\{a \otimes b \mid a \in \theta(A)^{\perp}, b \in \theta(B)^{\perp}\right\}^{\perp}$

\section{Facts:}

- It is an easy consequence of the definition that $(\theta A)^{\perp}=\theta A^{\perp}$ for any formula $A$.

- For any formula $A, \theta(A) \subseteq \operatorname{End}(\llbracket A \rrbracket)$.

\section{Interpretation of Proofs:}

We define the MGoI interpretation for proofs of MLL without the neutral elements, similarly to [18]. Every MLL sequent will be of the form $\vdash[\Delta], \Gamma$ where $\Gamma$ is a sequence of formulas and $\Delta$ is a sequence of cut formulas that have already been made in the proof of $\vdash \Gamma$ (see $[13,18]$ ). This device is used to keep track of the cuts in a proof of $\vdash \Gamma$. A proof $\Pi$ of $\vdash[\Delta], \Gamma$ is represented by a morphism $\llbracket \Pi \rrbracket \in \operatorname{End}(\otimes \llbracket \Gamma \rrbracket \otimes \llbracket \Delta \rrbracket)$. With $\Gamma=A_{1}, \cdots, A_{n}, \otimes \llbracket \Gamma \rrbracket$ stands for $\llbracket A_{1} \rrbracket \otimes \cdots \otimes \llbracket A_{n} \rrbracket$, similarly for $\Delta$. We drop the double brackets wherever there is no danger of confusion. We also define $\sigma=s \otimes \cdots \otimes s$ ( $m$-copies) where $s$ is the symmetry map at different types (omitted for convenience), and $|\Delta|=2 \mathrm{~m}$. The morphism $\sigma$ represents the cuts in the proof of $\vdash \Gamma$, i.e. it models $\Delta$. In the case where $\Delta$ is empty (that is for a cut-free proof), we define $\sigma: I \rightarrow I$ to be $1_{I}$ where $I$ is the unit of the monoidal product in $\mathbb{C}$.

Let $\Pi$ be a proof of $\vdash[\Delta], \Gamma$. We define the MGoI interpretation of $\Pi$, denoted by $\llbracket \Pi \rrbracket$, by induction on the length of the proof as follows.

1. $\Pi$ is an axiom $\vdash A, A^{\perp}, \llbracket \Pi \rrbracket:=s_{V, V}$ where $\llbracket A \rrbracket=\llbracket A^{\perp} \rrbracket=V$.

2. $\Pi$ is obtained using the cut rule on $\Pi^{\prime}$ and $\Pi^{\prime \prime}$ that is

$$
\begin{array}{cc}
\Pi^{\prime} & \Pi^{\prime \prime} \\
\vdots & \vdots \\
\frac{\vdash\left[\Delta^{\prime}\right], \Gamma^{\prime}, A \vdash\left[\Delta^{\prime \prime}\right], A^{\perp}, \Gamma^{\prime \prime}}{\vdash\left[\Delta^{\prime}, \Delta^{\prime \prime}, A, A^{\perp}\right], \Gamma^{\prime}, \Gamma^{\prime \prime}}(\text { cut })
\end{array}
$$

Define $\llbracket \Pi \rrbracket$ as follows: $\llbracket \Pi \rrbracket=\tau^{-1}\left(\llbracket \Pi^{\prime} \rrbracket \otimes \llbracket \Pi^{\prime \prime} \rrbracket\right) \tau$, where $\tau: \Gamma^{\prime} \otimes \Gamma^{\prime \prime} \otimes$ $\Delta^{\prime} \otimes \Delta^{\prime \prime} \otimes A \otimes A^{\perp} \rightarrow \Gamma^{\prime} \otimes A \otimes \Delta^{\prime} \otimes A^{\perp} \otimes \Gamma^{\prime \prime} \otimes \Delta^{\prime \prime}$ is a permutation, (double brackets and $\otimes$ are dropped for the sake of readability.) 
3. $\Pi$ is obtained using the exchange rule on the formulas $A_{i}$ and $A_{i+1}$ in $\Gamma^{\prime}$. That is $\Pi$ is of the form

$$
\begin{gathered}
\Pi^{\prime} \\
\vdots \\
\frac{\vdash[\Delta], \Gamma^{\prime}}{\vdash[\Delta], \Gamma} \text { (exchange) }
\end{gathered}
$$

where $\Gamma^{\prime}=\Gamma_{1}^{\prime}, A_{i}, A_{i+1}, \Gamma_{2}^{\prime}$ and $\Gamma=\Gamma_{1}^{\prime}, A_{i+1}, A_{i}, \Gamma_{2}^{\prime}$. Then, $\llbracket \Pi \rrbracket$ is obtained from $\llbracket \Pi^{\prime} \rrbracket$ by interchanging the rows $i$ and $i+1$. So, $\llbracket \Pi \rrbracket=\tau^{-1} \llbracket \Pi^{\prime} \rrbracket \tau$, where $\tau=1_{\Gamma_{1}^{\prime}} \otimes s \otimes 1_{\Gamma_{2}^{\prime} \otimes \Delta}$.

4. $\Pi$ is obtained using an application of the par rule, that is $\Pi$ is of the form:

$$
\begin{gathered}
\Pi^{\prime} \\
\vdots \\
\frac{\vdash[\Delta], \Gamma^{\prime}, A, B}{\vdash[\Delta], \Gamma^{\prime}, A>8 B}(\text { \& }) . \text { Then } \llbracket \Pi \rrbracket=\llbracket \Pi^{\prime} \rrbracket .
\end{gathered}
$$

5. $\Pi$ is obtained using an application of the times rule, that is $\Pi$ is of the form

$$
\begin{array}{ccc}
\Pi^{\prime} & \Pi^{\prime \prime} \\
\vdots & \vdots & \vdots \\
& \vdash\left[\Delta^{\prime}\right], \Gamma^{\prime}, A \vdash\left[\Delta^{\prime \prime}\right], \Gamma^{\prime \prime}, B \\
\frac{\vdash\left[\Delta^{\prime}, \Delta^{\prime \prime}\right], \Gamma^{\prime}, \Gamma^{\prime \prime}, A \otimes B}{\left.\llbracket \Pi^{\prime \prime} \rrbracket\right) \tau,} \text { (times) } & \text { Then } \llbracket \Pi \rrbracket=\tau^{-1}\left(\llbracket \Pi^{\prime} \rrbracket \otimes\right.
\end{array}
$$

where $\tau: \Gamma^{\prime} \otimes \Gamma^{\prime \prime} \otimes A \otimes B \otimes \Delta^{\prime} \otimes \Delta^{\prime \prime} \rightarrow \Gamma^{\prime} \otimes A \otimes \Delta^{\prime} \otimes \Gamma^{\prime \prime} \otimes B \otimes \Delta^{\prime \prime}$ is a permutation, (double brackets and $\otimes$ are dropped for the sake of readability.) This corresponds exactly to the definition of tensor product in Abramsky's $\mathcal{G}(\mathbb{C})($ see $[1,15]$.

Example 1. (a) Let $\Pi$ be the following proof:

$$
\frac{\vdash A, A^{\perp} \quad \vdash A, A^{\perp}}{\vdash\left[A^{\perp}, A\right], A, A^{\perp}}(\mathrm{cut})
$$

Then the MGoI semantics of this proof is given by

$$
\llbracket \Pi \rrbracket=\tau^{-1}(s \otimes s) \tau=s_{V \otimes V, V \otimes V}
$$

where $\tau=(1 \otimes 1 \otimes s)(1 \otimes s \otimes 1)$ and $\llbracket A \rrbracket=\llbracket A^{\perp} \rrbracket=V$.

(b) Now consider the following proof

$$
\begin{aligned}
& \frac{\vdash B, B^{\perp} \vdash C, C^{\perp}}{\vdash B, C, B^{\perp} \otimes C^{\perp}} \\
& \vdash B, B^{\perp} \otimes C^{\perp}, C \\
& \frac{\overline{\vdash B^{\perp} \otimes C^{\perp}, B, C}}{\vdash B^{\perp} \otimes C^{\perp}, B>8 C} \text {. }
\end{aligned}
$$


Its denotation is given by $s_{V \otimes W, V \otimes W}$ where $\llbracket B \rrbracket=\llbracket B^{\perp} \rrbracket=V$ and $\llbracket C \rrbracket=$ $\llbracket C^{\perp} \rrbracket=W$.

Proposition 3. Let $\Pi$ be an $M L L$ proof of $\vdash[\Delta], \Gamma$ where $|\Delta|=2 m$ and $|\Gamma|=$ $n$ (counting the occurrences of propositional variables), then $\llbracket \Pi \rrbracket$ is a fixed-point free involutive permutation on $n+2 m$ objects of $\mathbb{C}$. That is $\llbracket \Pi \rrbracket: V_{1} \otimes \cdots \otimes$ $V_{n+2 m} \rightarrow V_{1} \otimes \cdots \otimes V_{n+2 m}$ induces a permutation $\pi$ on $\{1,2 \cdots, n+2 m\}$ such that

$-\pi^{2}=1$

- For all $i \in\{1,2, \cdots, n+2 m\}, \pi(i) \neq i$.

- For all $i \in\{1,2, \cdots, n+2 m\}, V_{i}=V_{\pi(i)}$.

\subsection{Dynamics}

Dynamics is at the heart of the GoI interpretation as compared to denotational semantics and it is hidden in the cut-elimination process. The mathematical model of cut-elimination is given by the so called execution formula defined as follows:

$$
E X(\llbracket \Pi \rrbracket, \sigma)=\operatorname{Tr}_{\otimes \Gamma, \otimes \Gamma}^{\otimes \Delta}((1 \otimes \sigma) \llbracket \Pi \rrbracket)
$$

where $\Pi$ is a proof of the sequent $\vdash[\Delta], \Gamma$, and $\sigma=s \otimes \cdots \otimes s$ ( $m$ times) models $\Delta$.

Note that $E X(\llbracket \Pi \rrbracket, \sigma)$ is a morphism from $\otimes \Gamma \rightarrow \otimes \Gamma$, when it exists. We shall prove (see Theorem 2 below) that the execution formula always exists for any MLL proof $\Pi$.

Example 2. Consider the proof $\Pi$ in Example 1 above. Recall also that $\sigma=s$ in this case $(m=1)$. Then $E X(\llbracket \Pi \rrbracket, \sigma)=\operatorname{Tr}\left(\left(1 \otimes s_{V, V}\right) s_{V \otimes V, V \otimes V}\right)=s_{V, V}$.

Note that in this case we have obtained the MGoI interpretation of the cutfree proof $\vdash A, A^{\perp}$, obtained by applying Gentzen's Hauptsatz to the proof $\Pi$.

\section{Soundness of the Interpretation}

In this section we shall prove one of the main results of this paper: the soundness of the MGoI interpretation. We show that if a proof $\Pi$ is reduced (via cutelimination) to another proof $\Pi^{\prime}$, then $E X(\llbracket \Pi \rrbracket, \sigma)=E X\left(\llbracket \Pi^{\prime} \rrbracket, \tau\right)$; that is, $E X(\llbracket \Pi \rrbracket, \sigma)$ is an invariant of reduction. In particular, if $\Pi^{\prime}$ is cut-free (i.e. a normal form) we have $E X(\llbracket \Pi \rrbracket, \sigma)=\llbracket \Pi^{\prime} \rrbracket$. Intuitively this says that if one thinks of cut-elimination as computation then $\llbracket \Pi \rrbracket$ can be thought of as an algorithm. The computation takes place as follows: if $\operatorname{EX}(\llbracket \Pi \rrbracket, \sigma)$ exists then it yields a datum (cf. cut-free proof). This intuition will be made precise below (see Theorems 2,3). 
Lemma 4 (Associativity of cut). Let $\Pi$ be a proof of $\vdash[\Gamma, \Delta], \Lambda$ and $\sigma$ and $\tau$ be the morphisms representing the cut-formulas in $\Gamma$ and $\Delta$ respectively. Then $E X(\llbracket \Pi \rrbracket, \sigma \otimes \tau)=E X(E X(\llbracket \Pi \rrbracket, \tau), \sigma)=E X(E X((1 \otimes s) \llbracket \Pi \rrbracket(1 \otimes s), \sigma), \tau)$, whenever all traces exist.

Definition 4. Let $\Gamma=A_{1}, \cdots, A_{n}$ and $V_{i}=\llbracket A_{i} \rrbracket$. A datum of type $\theta \Gamma$ is a morphism

$M: \otimes_{i} V_{i} \rightarrow \otimes_{i} V_{i}$ such that for any $\beta_{i} \in \theta\left(A_{i}^{\perp}\right), \otimes_{i} \beta_{i} \perp M$, and $M \cdot \beta_{1}:=$ $\operatorname{Tr}^{V_{1}}\left(s_{\otimes_{i} V_{i}, V_{1}}\left(\beta_{1} \otimes 1_{V_{2}} \otimes \cdots \otimes 1_{V_{n}}\right) M s_{\otimes_{i} V_{i}, V_{1}}\right)$ exists. (In Girard's notation [13], $M \cdot \beta_{1}$ corresponds to ex $\left.\left(C U T\left(\beta_{1}, M\right)\right)\right)$.

$A n$ algorithm of type $\theta \Gamma$ is a morphism $M: \otimes_{i} V_{i} \otimes \llbracket \Delta \rrbracket \rightarrow \otimes_{i} V_{i} \otimes \llbracket \Delta \rrbracket$ for some $\Delta=B_{1}, B_{2}, \cdots, B_{2 m}$ with $m$ a nonnegative integer and $B_{i+1}=B_{i}^{\perp}$ for $i=1 \cdots, 2 m-1$ such that for $\sigma: \otimes_{j=1}^{2 m} \llbracket B_{j} \rrbracket \rightarrow \otimes_{j=1}^{2 m} \llbracket B_{j} \rrbracket$ defined by $\otimes_{j=1}^{2 m-1} s \llbracket B_{j} \rrbracket, \llbracket B_{j+1} \rrbracket, E X(M, \sigma)$ exists and is a datum of type $\theta \Gamma . \sigma$ is defined to be $1_{I}$ for $m=0$.

Lemma 5. Let $\Gamma=A_{2}, \cdots, A_{n}, V_{i}=\llbracket A_{i} \rrbracket$, and $M: \otimes_{i} V_{i} \rightarrow \otimes_{i} V_{i}$, for $i=$ $1, \cdots, n$. Then, $M$ is a datum of type $\theta\left(A_{1}, \Gamma\right)$ iff for every $a_{1} \in \theta\left(A_{1}^{\perp}\right), M \cdot a_{1}$ (defined as above) exists and is in $\theta(\Gamma)$.

Theorem 2 (Proofs as algorithms). Let $\Gamma$ be an $M L L$ sequent, and $\Pi$ be a proof of $\vdash[\Delta], \Gamma$. Then $\llbracket \Pi \rrbracket$ is an algorithm of type $\theta \Gamma$.

Corollary 1 (Existence of Dynamics). Let $\Pi$ be an $M L L$ proof of a sequent $\vdash[\Delta], \Gamma$. Then $\operatorname{Ex}(\llbracket \Pi \rrbracket, \sigma)$ exists.

Theorem 3 (EX is an invariant). Let $\Pi$ be a proof of a sequent $\vdash[\Delta], \Gamma$ in $M L L$. Then,

- If $\Pi$ reduces to $\Pi{ }^{\prime}$ by any sequence of cut-eliminations, then $E X(\llbracket \Pi \rrbracket, \sigma)=$ $E X\left(\llbracket \Pi^{\prime} \rrbracket, \tau\right)$. So $E X(\llbracket \Pi \rrbracket, \sigma)$ is an invariant of reduction.

- In particular, if $\Pi^{\prime}$ is any cut-free proof obtained from $\Pi$ by cut-elimination, then $E X(\llbracket \Pi \rrbracket, \sigma)=\llbracket \Pi^{\prime} \rrbracket$.

\section{Completeness}

In this section we prove a completeness theorem for MLL in a partially traced category equipped with an orthogonality relation, under MGoI semantics. Motivated by this result we prove a completeness theorem for MLL in any traced Unique Decomposition Category with a reflexive object, under (uniobject) GoI semantics [18], which we present in the next section.

Theorem 4 (Completeness). Let $M$ be a fixed-point free involutive permutation from $V_{1} \otimes \cdots \otimes V_{n} \rightarrow V_{1} \otimes \cdots \otimes V_{n}$ (induced by a permutation $\mu$ on $\{1,2, \cdots, n\}$ ) where $n>0$ is an even integer, $V_{i}=\llbracket A_{i} \rrbracket$, and $V_{i}=V_{\mu(i)}$ for all $i=1, \cdots, n$. Then there is a provable $M L L$ formula $\varphi$ built from the $A_{i}$, with a proof $\Pi$ such that $\llbracket \Pi \rrbracket=M$. 


\section{Completeness For Uni-GoI Semantics}

The above theorem may be applied to give a completeness theorem for the untyped (i.e. uni-object) GoI semantics for MLL, as discussed in our papers [18, 19]. The theorem below is related to work of Danos and Regnier. We hope in future work to detail these connections.

In what follows we consider variables $j_{1}, j_{2}, k_{1}, k_{2}$ which are the components of the retraction $U \otimes U \triangleleft U(j, k)$ where $U$ is a reflexive object in a traced UDC $\mathbb{C}$ (see [18] for details about GoI semantics in UDCs.) In Girard's terminology [13] $j_{1}=p, j_{2}=q, k_{1}=p^{*}, k_{2}=q^{*}$. We define ( $)^{*}$ on words built on the alphabet $\Sigma=\left\{0,1, j_{1}, j_{2}, k_{1}, k_{2}\right\}$ as follows: $0^{*}=0,1^{*}=1, j_{1}^{*}=k_{1}, j_{2}^{*}=k_{2}, k_{1}^{*}=j_{1}$ and $k_{2}^{*}=j_{2} .1$ is the neutral element and 0 the null element (annihilator) for juxtaposition. $(w \alpha)^{*}=\alpha^{*} w^{*}$ for $w$ a word on $\Sigma$ and $\alpha \in \Sigma$. Note that words $w$ on $\Sigma$ cannot have $k$ 's occurring after $j$ 's because of equations $k_{1} j_{1}=k_{2} j_{2}=1$ and $k_{2} j_{1}=k_{1} j_{2}=0$. Thus words $w$ on $\Sigma$ are of the form $w=u v$ where $v$ is a word on $\Sigma$ consisting of $k_{1}, k_{2}$ only and $u$ a word on $\Sigma$ consisting of $j_{1}, j_{2}$ only, either $u$ or $v$ can be 1 . We call $v$ the $k$-part and $u$, the $j$-part of $w$. For example, the $k$ part of $j_{1} j_{2} k_{2} k_{1}^{2}$ is $k_{2} k_{1}^{2}$ and its $j$-part is $j_{1} j_{2}$.

Theorem 5. Consider the polynomial $\varphi \in \mathbb{F}_{2}\left[j_{1}, j_{2}, k_{1}, k_{2}\right]$ where $\mathbb{F}_{2}$ is the finite field of characteristic 2. Then, $\varphi$ is the denotation of an MLL proof iff

- $\varphi$ has an even number $n$ of summands,

- For each summand $w, w^{2}=0$ and $w^{*}$ is a summand in $\varphi$,

- No two summands have identical k-parts.

Lemma 6. Let $\Gamma$ be an MLL sequent of length (counting the occurrences of propositional variables) $n$ and $\Pi$ be a proof of $\vdash \Gamma$, then $\llbracket \Pi \rrbracket=J \llbracket \Pi \rrbracket{ }_{M} K$, where $\llbracket \Pi \rrbracket$ and $\llbracket \Pi \rrbracket_{M}$ are the $G o I$ and $M G o I$ interpretations of $\Pi$, respectively, $J: U^{n} \rightarrow U$ is a morphism built from $j$ and $1_{U}$ by tensoring and composition, and $K=J^{*}$.

\section{Conclusion and Future Work}

In this work we introduce a new semantics called multiobject Geometry of Interaction (MGoI). The definition of this semantics, while inspired by GoI, differs from it in many significant points. Namely, we deal with many objects in the underlying category, we make use of a denotational semantics to define the interpretation for the formulas and the execution formula is based on partial traces and trace ideals. Moreover, the orthogonality relation is linked to the notion of trace class. We are thus able to carry out our interpretation in the categories of finite dimensional vector spaces and the other examples of trace ideal mentioned above. This is not possible for the earlier theory of uniobject GoI as none of these categories have non-trivial reflexive objects. We are currently working 
on interpreting our MGoI on Banach spaces and related categories, by finding appropariate classes of trace ideals.

An obvious direction for future research is to extend our interpretation to the exponentials and additives. Also there are many new directions that open up due to the introduction of partial trace and abstract orthogonality into the world of GoI semantics. In particular, we shall pursue further research along the correspondence of trace class/nuclear morphisms as achieved in [2] for their examples. The notion of nuclearity, intuitively captures the notion of finiteness, for example every finite dimensional vector space is nuclear, but this is with respect to the tensor product of vector spaces as the monoidal product. Our monoidal product is the direct sum and it would be very illuminating to know what the nuclear objects and morphisms are and what is being characterized by them. In particular, are they characterizing a class of proof denotations, etc.?

Another point of interest is to further probe the trace class/orthogonality interplay. In particular, we have shown that the orthogonality relations we deal with can all be nicely characterized as trace class morphisms, and that this defined orthogonality satisfies Hyland-Schalk axioms. It is natural to seek examples of traces that are induced by other notions of orthogonalities, especially those arising in functional analysis. We hope this may lead to new classes of GoI models, perhaps connected to current work in operator algebras.

\section{References}

1. Abramsky, S. (1996), Retracing Some Paths in Process Algebra. In CONCUR 96, Springer LNCS 1119, 1-17.

2. Abramsky, S., Blute, R. and Panangaden, P. (1999), Nuclear and trace ideals in tensored *-categories, J. Pure and Applied Algebra vol. 143, 3-47.

3. Abramsky, S., Haghverdi, E. and Scott, P.J. (2002), Geometry of Interaction and Linear Combinatory Algebras. MSCS, vol. 12(5), 2002, 625-665, CUP.

4. Abramsky S. and Jagadeesan, R. (1994), Games and full completeness for multiplicative linear logic. J. Symbolic Logic 59(2), pp. 543-574.

5. Abramsky, S. and Jagadeesan, R. (1994), New Foundations for the Geometry of Interaction. Information and Computation 111 (1), 53-119.

6. Baillot, P. (1995), Abramsky-Jagadeesan-Malacaria strategies and the geometry of interaction, mémoire de DEA, Universite Paris 7, 1995.

7. Baillot, P. and Pedicini, M. (2000), Elementary complexity and geometry of interaction, Fundamenta Informaticae vol. 45, dno 1-2, 2001

8. Danos, V. (1990), La logique linéaire appliquée à l'étude de divers processus de normalisation et principalement $d u \lambda$-calcul. $\mathrm{PhD}$ thesis, Université Paris VII.

9. Danos, V. and Regnier, L. (1995), Proof-nets and the Hilbert Space. In: Advances in Linear Logic, London Math. Soc. Notes, 222, CUP, 307-328.

10. Gonthier, G., Abadi, M. and Lévy, J.-J. (1992), The geometry of optimal lambda reduction. In Proceedings of Logic in Computer Science, vol. 9 pp. 15-26.

11. Girard, J.-Y. (1987), Linear Logic. Theoretical Computer Science 50 (1), pp. 1-102.

12. Girard, J.-Y. (1988), Geometry of Interaction II: Deadlock-free Algorithms. In Proc. of COLOG'88, LNCS 417, Springer, 76-93.

13. Girard, J.-Y. (1989a) Geometry of Interaction I: Interpretation of System F. In Proc. Logic Colloquium 88, North Holland, 221-260. 
14. Girard, J.-Y. (1995), Geometry of Interaction III: Accommodating the Additives. In: Advances in Linear Logic, LNS 222,CUP, 329-389,

15. Haghverdi, E. A Categorical Approach to Linear Logic, Geometry of Proofs and Full Completeness, PhD Thesis, University of Ottawa, Canada 2000.

16. Haghverdi, E. Unique Decomposition Categories, Geometry of Interaction and combinatory logic, Math. Struct. in Comp. Science, vol. 10, 2000, 205-231.

17. Haghverdi E., Partially Additive Categories and Fully Complete Models of Linear Logic, TLCA'01, Springer LNCS, vol. 2044, pp. 197-216, 2001.

18. Haghverdi, E. and P.J.Scott. A categorical model for the Geometry of Interaction, to appear in Theoretical Computer Science (cf. ICALP 2004, Springer LNCS 3142).

19. Haghverdi, E. and P.J.Scott. From Geometry of Interaction to Denotational Semantics, CTCS2004. In ENTCS, Elsevier. ).

20. Hasegawa, M. (1997), Recursion from Cyclic Sharing : Traced Monoidal Categories and Models of Cyclic Lambda Calculus, Springer LNCS 1210, 196-213.

21. Hines, P. A categorical framework for finite state machines, Math. Struct. in Comp. Science, vol. 13, 2003, 451-480.

22. Hyland, M and Schalk, A. Glueing and Orthogonality for Models of Linear Logic. Theoretical Computer Science vol. 294 2003, pp. 183-231.

23. Jeffrey, A.S.A., Premonoidal categories and a graphical view of programs, available from http://klee.cs.depaul.edu/premon/, 1998.

24. Joyal, A., Street, R. and Verity, D. (1996), Traced Monoidal Categories. Math. Proc. Camb. Phil. Soc. 119, 447-468.

25. Laurent, O., A Token Machine for Full Geometry of Interaction. In TLCA '01, SLNCS 2044, pp. 283-297.

26. Mac Lane, S. (1998), Categories for the Working Mathematician, 2nd Ed. Springer.

27. Malacaria, P. and Regnier. L. (1991), Some Results on the Interpretation of $\lambda$ calculus in Operator Algebras. In Proc. LICS (1991), 63-72, IEEE Computer Society Press.

28. Plotkin, G. Trace Ideals, MFPS 2003 invited lecture, Montreal.

29. Regnier, L. (1992), Lambda-calcul et Réseaux, PhD Thesis, Université Paris VII. 